\title{
The impact of visual illusions on perception, action planning, and motor performance
}

\author{
Greg Wood • Samuel J. Vine • Mark R. Wilson
}

Published online: 12 June 2013

(C) Psychonomic Society, Inc. 2013

\begin{abstract}
The present study extended recent research revealing that illusions can influence performance in golf putting (Witt, Linkenauger, \& Proffitt Psychological Science, 23, 397-399, 2012), by exploring the potential mediating roles of attention and action planning. Glover and Dixon's (Journal of Experimental Psychology. Human Perception and Performance, 27, 560-572, 2001) planning-control model suggests that both perceptual and movement-planning processes are prone to illusion-based bias. We therefore predicted that both the perception of target size and a measure of attentional control related to movement planning in golf putting (the quiet eye) would be influenced by the illusion. Moreover, as performance could not be corrected using online control (once the ball was struck), we predicted that these biases would also influence performance. We therefore proposed a three-stage process by which illusory context biases perceptual processes, which in turn bias subsequent attentional control related to movement planning, which in turn biases motor performance. Forty novice golfers completed an Ebbinghaus illusion putting task that was designed to manipulate their perceptions of target size, while quiet eye duration and performance (mean radial error) were measured. The results indicated that the illusion was effective in facilitating differences in perceived target size, with perceptually bigger holes promoting longer quiet eye durations and more accurate putting. Follow-up mediation analyses revealed that illusion-based differences in size perception partially mediated illusion-based differences in both quiet eye duration and performance. Moreover, the relationship between illusion-based differences in quiet eye duration and performance was also significant. Future research should further test this three-stage process of bias in other far-aiming tasks in which online control cannot be used.
\end{abstract}

G. Wood $(\varangle) \cdot$ S. J. Vine $\cdot$ M. R. Wilson

College of Life and Environmental Sciences, Richards Building, University of Exeter, St Luke's Campus, Exeter EX1 2LU, UK

e-mail: g.wood@exeter.ac.uk
Keywords Titchener circles · Quiet eye $\cdot$ Size perception . Movement planning · Online control

Illusions provide an interesting means to examine the larger issue of how the central nervous system codes and uses visual information for different types of cognitive and motor tasks (Mendoza, Hansen, Glazebrook, Keetch, \& Elliott, 2005). Recently, Witt and colleagues explored the impact of an illusory visual context on golf-putting performance (Witt, Linkenhauger, \& Proffitt, 2012). Specifically, an Ebbinghaus illusion around the target hole (see Fig. 1) was effective at inducing significant differences in perceptions of target size and in subsequent putting performance: Participants holed significantly more putts when they were putting to a perceptually bigger hole. The aim of the present study was to elucidate potential mechanisms underpinning the illusion effect shown by Witt et al., using Glover and Dixon's (2001) planning-control model as a theoretical framework. We hoped that such research might help further our understanding of how perceptual and attentional processes mediate performance variability in far-aiming tasks like golf putting.

Glover and Dixon's (2001) model posits that the extent to which illusory bias influences a goal-directed action will depend on the relative importance of advanced planning and online control in the realization of that action. Specifically, illusions affect the planning of actions but do not affect their online control, so that errors in planning (caused by the illusory context surrounding a target) can be corrected online as the movement unfolds (via visual and proprioceptive feedback; Glover, 2002). For example, Glover and Dixon found that the Ebbinghaus illusion had a large effect on grip aperture early in the movement of a grasping task, but that this effect dissipated as the hand approached the target. However, when online control cannot regulate an unfolding action - for instance, in memory-guided movements - the impact of biased preplanning processes may be realized (Mendoza et al., 2005). 
Golf putting provides an excellent example of a memoryguided movement, since performers must hold target-related information in working memory while fixating a ball that is propelled toward the unfixated target hole (Vickers, 1992, 2007). Vickers (1992) provided a detailed examination of the gaze behavior of expert and less expert golfers as they putted to a hole from $3 \mathrm{~m}$ away. The only gaze measure that differentiated superior performance was the duration of the final fixation on the ball, which the experts maintained for longer throughout the putting stroke. Vickers subsequently described this fixation as the "quiet eye"- the duration of the final fixation toward a relevant target before the initiation of a critical movement (Vickers, 1996, 2007). The quiet eye has been proposed to facilitate a period of preprogramming of the critical parameters of the goal-directed action (e.g., force and direction), and it characterizes superior performance in a range of far-aiming tasks (Mann, Williams, Ward, \& Janelle, 2007). Recent work by Mann and colleagues has supported this contention, identifying strong associations between the quiet eye and a neural marker of motor planning - the Bereitschaftspotential (Mann, Coombes, Mousseau, \& Janelle, 2011; Vine, Moore, \& Wilson, 2013b). This conceptualization of the quiet eye as an objective measure of motor planning resonates with Glover's (2004) postulation that the planning system's role is to determine the initial kinematic parameterization of movements and then to select and run this motor program.

The aim of the present study was therefore to extend Witt et al.'s (2012) study, by measuring not only indices of perception and performance, but also a measure of goal-directed planning that is relevant to Glover and Dixon's (2001) planning-control model. We hypothesized that the Ebbinghaus illusion putting task would bias both the perceptual and planning systems, influencing perceptions of target size, memory-guided planning (quiet eye), and-because biases could not be modified once the ball was moving - performance (radial error). We further examined our exploratory proposal for a three-stage relationship between biases in perception, subsequent planning, and final performance, via mediation analyses.

\section{Method}

\section{Participants}

A group of 40 participants ( 30 male, 10 female) volunteered to take part in the study. The participants (mean age 20.85years, $S D=3.18$ ) were classed as novices and had minimal formal golfing experience (mean of 1.98 years, $S D=3.93$ ). The testing procedures were approved by a local ethics committee and all participants gave written consent after the procedures were fully explained.
The Ebbinghaus putting task

The illusions were created in Microsoft PowerPoint and displayed on an artificial putting green (length $=6 \mathrm{~m}$, width $=$ $2.5 \mathrm{~m}$ ) using a downward-facing projector (Hitachi XGA, CP$\mathrm{X} 275$ ). When projected, each illusion consisted of a central 5$\mathrm{cm}$ target hole that was surrounded by either 12 small $(3.3-\mathrm{cm}$ diameter) or five large $(29.2-\mathrm{cm}$ diameter) flanker circles to create the illusion effect (Fig. 1). As previous research had shown that the distance between the central target and the flankers' edges (the dotted lines in Fig. 1) can confound the illusion effect (Roberts, Harris, \& Yates, 2005), this was standardized to $23.9 \mathrm{~cm}$ across the illusions. ${ }^{1}$

Each illusion was presented in a ten-trial block, and each block of trials consisted of the same illusion presented consecutively, $15 \mathrm{~cm}$ to either the left or right of the center of the display. This ensured that participants had to focus on and realign to the "new" target after each shot. It also minimized trial-to-trial learning effects that could have influenced our results. The putting task required participants to putt from a distance of $1.75 \mathrm{~m}$ using a standard-length $(90 \mathrm{~cm})$ golf putter (Sedona 2, Ping, Phoenix, AZ) and regular-sized (diameter $=4.27 \mathrm{~cm})$ white golf balls.

\section{Measures}

Perceptions of target size Judgments of hole size were obtained by asking participants to draw life-sized replicas of each target hole on a laptop loaded with PowerPoint presentation software, situated on a table in front of their putting position (i.e., at the putting distance, $1.75 \mathrm{~m}$ ). These size estimates were taken at the beginning of each block of ten putts in each illusion condition. When the vertical and horizontal measurements of circle size differed (i.e., an ellipse was drawn), a mean perceived size was calculated.

Performance The medial radial error (the distance in centimeters from the final ball position to the nearest edge of the hole) was recorded for each putt, and a mean value for each condition computed. A putt that landed inside or broke the projected line of the hole was recorded as " 0 " (Moore, Vine, Cooke, Ring, \& Wilson, 2012).

Quiet eye Each participant was fitted with an Applied Science Laboratories (Bedford, MA) Mobile Eye gaze registration system, which measured the momentary point of gaze at $30 \mathrm{~Hz}$. The quiet eye was operationally defined for golf putting as the final fixation toward the ball prior to the initiation of the backswing, and its duration as the time between

\footnotetext{
${ }^{1}$ Note that Witt et al. (2012) did not make this adjustment in their study. Also, we did not adopt the additional 10 -cm-diameter target used by Witt et al., as this did not reveal an illusion bias in that study.
} 


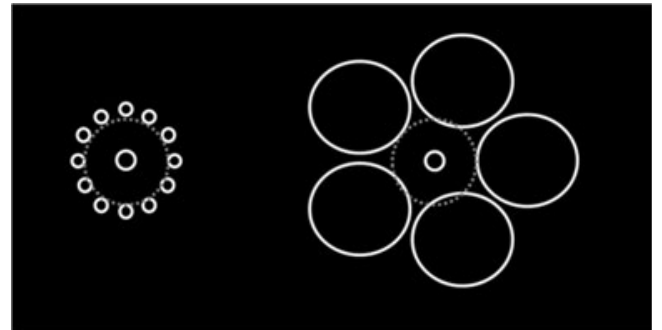

Fig. 1 Our Ebbinghaus illusion for the perceptually bigger (left) and perceptually smaller (right) holes. The graydotted lines represent the standardization of the distance between the hole and the flanker edges

the onset and offset of this fixation (as in Moore et al., 2012; Vickers, 2007; Vine, Lee, Moore, \& Wilson, 2013a; Wilson \& Pearcey, 2009). Quiet eye onset occurred before the backswing began, and quiet eye offset occurred when the gaze deviated off the ball location by $1^{\circ}$ or more for greater than $100 \mathrm{~ms}$. The interrater reliability was $91.8 \%$.

Procedure

Participants attended individually, and the eyetracker was calibrated whilst they stood in their normal putting stance. Participants were then presented with a 5-cm hole that was flanked by 12 holes of the same diameter, to which they took ten practice putts. This initial practice session gave participants putting practice to a flanked target in order to minimize potential learning effects. Prior to taking these practice putts, it was explained to participants that soon after they had made contact with the ball, the target would disappear. When this happened, they were required to turn around and face an adjacent wall. An experimenter advanced the presentation to a blank slide (making the target disappear) once the ball had passed a critical point $30 \mathrm{~cm}$ from the original putting position. This prevented any knowledge of a participant's putting performance from influencing subsequent size judgments (Lee, Lee, Carello, \& Turvey, 2012) and performance (a potential confound in Witt et al.'s, 2012, study). Whilst the participant's back was turned, the illusion was redisplayed and measurements of radial error were taken using a tape measure. After these measurements were taken, the ball was returned to the putting position, the presentation was advanced to the next illusion stimulus, and participants were signaled to turn around and take their next putt. After this initial block of practice trials, the illusion conditions (ten putts) were presented in a counterbalanced fashion using exactly the same procedure.

Data analysis

Illusion-based differences in the variables of interest were subjected to paired-samples $t$ tests, with effect sizes calculated using Cohen's $d$. To examine whether condition-based differences in perceived hole size and quiet eye mediated differences in performance, the difference/sum regression mediation procedure for repeated measures designs was applied (Judd, Kenny, \& McClelland, 2001).

\section{Results}

Condition-related differences in the dependent variables were first computed (see Table 1 for the results of these paired-samples $t$ tests). Relative to the perceptually larger hole condition, in the perceptually smaller hole condition the radial error was $3.64 \mathrm{~cm}$ greater, the target was perceived as being $4.5 \mathrm{~mm}$ smaller, and the quiet eye duration was $170 \mathrm{~ms}$ shorter. Because we observed significant illusion effects for all measures, the prior conditions for follow-up mediation analyses were satisfied (Judd et al., 2001).

\section{Perceived target size and radial error}

The regression analysis revealed that illusion-based differences in a target's perceived size significantly predicted differences in radial error $\left(R^{2}=.18, b=-5.66\right), t(39)=$

Table 1 Mean (with $S D$ ) results for the dependent variables between illusion conditions, with paired-sample $t$-test statistics $(t$ statistic, degrees of freedom, $p$ value, and effect size [Cohen's $d]$ )

\begin{tabular}{|c|c|c|c|c|c|c|}
\hline Measure & Perceptually Bigger Hole & Perceptually Smaller Hole & $t$ & $d f$ & $p$ & $d$ \\
\hline Perceived hole size (mm) & $4.65(1.63)$ & $4.20(1.69)$ & 4.56 & 39 & .000 & 0.73 \\
\hline Performance error $(\mathrm{cm})$ & $26.58(12.33)$ & $30.21(13.76)$ & -2.51 & 39 & .017 & 0.40 \\
\hline Quiet eye duration (ms) & $1,651.08(708.35)$ & $1,481.24(748.76)$ & 2.78 & 30 & .009 & 0.50 \\
\hline
\end{tabular}

The reduced degrees of freedom for the quiet eye variables are due to lost or incomplete data from nine participants, due to problems with calibration or technical issues with the gaze registration system during testing 
$-2.92, p=.006$. The original difference in radial error of $3.64 \mathrm{~cm}$ was reduced to a nonsignificant residual difference of $1.61 \mathrm{~cm}$ when the effect of perceived size was accounted for, $t(39)=1.09, p=.283$, suggesting that perceived size was a partial mediator.

Perceived target size and quiet eye duration

The regression analysis revealed that illusion-based differences in perceived size significantly predicted differences in quiet eye duration $\left(R^{2}=.22, b=494.77\right), t(30)=2.88, p=.007$. The original difference in quiet eye duration of $170 \mathrm{~ms}$ was reduced to a nonsignificant residual difference of $57 \mathrm{~ms}$ when the effect of perceived size was accounted for, $t(30)=0.44, p=.661$, suggesting that perceived size was a partial mediator.

Quiet eye duration and radial error

The regression analysis revealed that illusion-based differences in quiet eye duration significantly predicted differences in radial error $\left(R^{2}=.14, b=-0.005\right), t(30)=-2.14$, $p=.041$. However, the residual difference of $3.64 \mathrm{~cm}$ remained unchanged when the effect of quiet eye duration was accounted for, and though the effect of the illusion on radial error was reduced, it was still significant, $t(30)=2.49$, $p=.019$.

\section{Discussion}

In this study, we sought to advance the theoretical understanding of how illusions influence the perceptual and motor processes underpinning memory-dependent goal-directed aiming. Specifically, we sought to extend Witt et al.'s (2012) research investigating the effect of illusions on golf-putting performance by testing the predictions of Glover and Dixon's (2001) planning-control model, using an objective measure of planning in golf putting (the quiet eye; Vickers, 1992, 2007). The planning-control model postulates that similar visual representations for perception and action planning take into account the context surrounding a target (Glover, 2002, 2004). We therefore predicted that the illusory context would bias both measures in the same direction. Additionally, as performance could not be adjusted via online control (which uses an independent representation; Glover, 2002), we predicted that performance would also be biased.

We found significant illusion-based biases in perceptions of target size $(\sim 4.5 \mathrm{~mm})$ and performance error $(\sim 3.7 \mathrm{~cm})$. Furthermore, additional mediation analyses suggested that differences in perceived size partially mediated the performance effect of the illusion. To further advance our understanding of the processes underpinning this relationship, we sought to test a three-stage model: Does goal-directed planning further mediate this perception-performance effect? Specifically, we predicted that biases in the perception of the target hole would influence subsequent motor planning (Glover, 2002) that occurs while the performer fixates the ball (Vickers, 1992), and that this, in turn, would bias performance (Moore et al., 2012; Vickers, 1992; Wilson \& Pearcey, 2009; Vine, Lee, et al., 2013a).

In support of the model, we found significant illusionbased biases in quiet eye duration $(170 \mathrm{~ms})$, with shorter quiet eye durations occurring during putting to the perceptually smaller hole. Additionally, the mediation analyses revealed that illusion-based differences in perceptions of target size were a significant predictor $(22 \%)$ and a partial mediator of the illusion-based effect on quiet eye duration. The final stage of the model was only partially upheld: Whereas differences in quiet eye duration significantly predicted changes in radial error, the partial mediation effect was not apparent. However, it is recognized that traditional, causal-step approaches to mediation - which had to be used in this study, due to the within-subjects design employed - can be more conservative than modern, bootstrapping procedures (Hayes, 2009).

A number of other potential explanations for the effects that we have demonstrated need to be considered. First, it is possible that other aspects of the Ebbinghaus illusion related to the visual context also influenced planning and performance, beyond effects related to the perception of size. For example, the 12 small flanking circles that created a perceptually bigger hole may also have created an additional "target zone" surrounding the hole - an effect that was missing or minimized with the five large circles (see Roberts et al., 2005, Fig. 1b). Indeed, although the 12 small circles created a more hittable Gestalt target zone (Lee et al., 2012), the five large circles may actually have distracted target fixations and disrupted efficient planning (Glover, 2002). Moreover, in the present study we were not able to clarify how potential psychological variables (e.g., confidence; Witt et al., 2012) might influence the relationship between illusion bias and subsequent performance. ${ }^{2}$

To conclude, the present study extends the findings of Witt et al. (2012) by discussing proposed mechanisms linking perceptual and motor biases, as outlined in Glover and Dixon's (2001) planning-control model. Both perceptual-cognitive judgments and movement-planning processes were biased by the visual context associated with the Ebbinghaus illusion, and these biases were associated with subsequent performance variability. We found partial support for our contention that this process might unfold over time in three stages: Illusions influence the perception of

\footnotetext{
$\overline{{ }^{2} \text { We measured }}$ reported self-confidence using Krane's (1994) Mental Readiness Form 3 prior to each block of putts. This single-item measure incorporates a 10-point Likert scale, anchored between 1 (not confident) and 11 (confident). However, no significant differences were uncovered, $t(39)=0.68, p=.496$, between the perceptually bigger (mean $=$ $4.04, S D=1.99)$ and perceptually smaller (mean $=3.93, S D=2.07)$ hole conditions.
} 
size, which influences subsequent memory-guided planning, which in turn influences far-aiming performance (in which online adjustments cannot be made). Future research should attempt to corroborate these predictions, not only in relation to effects related to illusory context, but also in other contexts where performance variability in visually guided tasks is experienced (e.g., pressure).

\section{References}

Glover, S. R. (2002). Visual illusions affect planning but not control. Trends in Cognitive Sciences, 6, 288-292.

Glover, S. R. (2004). Separate representations in the planning and control of action. The Behavioral and Brain Sciences, 27, 3-24. disc. 24-78.

Glover, S. R., \& Dixon, P. (2001). Dynamic illusion effects in a reaching task: Evidence for separate visual representations in the planning and control of reaching. Journal of Experimental Psychology. Human Perception and Performance, 27, 560-572. doi:10.1037/0096-1523.27.3.560

Hayes, A. F. (2009). Beyond Baron and Kenny: Statistical mediation analysis in the new millennium. Communication Monographs, 76, 408-420.

Judd, C. M., Kenny, D. A., \& McClelland, G. H. (2001). Estimating and testing mediation and moderation in withinsubject designs. Psychological Methods, 6, 115-134. doi:10.1037/ 1082-989X.6.2.115

Krane, V. (1994). The mental readiness form as a measure of competitive state anxiety. Sport Psychologist, 8, 189-202.

Lee, Y., Lee, S., Carello, C., \& Turvey, M. T. (2012). An archer's perceived form scales the "hitableness" of archery targets. Journal of Experimental Psychology. Human Perception and Performance, 38, 1125-1131. doi:10.1037/a0029036

Mann, D. T. Y., Coombes, S. A., Mousseau, M. B., \& Janelle, C. M. (2011). Quiet eye and the Bereitschaftspotential: Visuomotor mechanisms of expert motor performance. Cognitive Processing, 12, 223-234. doi:10.1007/s10339-011-0398-8

Mann, D. T. Y., Williams, A. M., Ward, P., \& Janelle, C. M. (2007). Perceptual-cognitive expertise in sport: A meta-analysis. Journal of Sport \& Exercise Psychology, 29, 457-478.

Mendoza, J., Hansen, S., Glazebrook, C. M., Keetch, K. M., \& Elliott, D. (2005). Visual illusions affect both movement planning and online control: A multiple cue position on bias and goal-directed action. Human Movement Science, 24, 760-773. doi:10.1016/ j.humov.2005.09.002

Moore, L. J., Vine, S. J., Cooke, A., Ring, C., \& Wilson, M. R. (2012). Quiet eye training expedites motor learning and aids performance under heightened anxiety: The roles of response programming and external attention. Psychophysiology, 49, 1005-1015. doi:10.1111/ j.1469-8986.2012.01379.x

Roberts, B., Harris, M. G., \& Yates, T. A. (2005). The role of inducer size and distance in the Ebbinghaus illusion (Titchener circles). Perception, 34, 847-856. doi:10.1068/p5273

Vickers, J. N. (1992). Gaze control in putting. Perception, 21, 117-132.

Vickers, J. N. (1996). Visual control when aiming at a far target. Journal of Experimental Psychology. Human Perception and Performance, 22, 342-354. doi:10.1037/0096-1523.22.2.342

Vickers, J. N. (2007). Perception, cognition and decision training: The quiet eye in action. Champaign, IL: Human Kinetics.

Vine, S. J., Lee, D., Moore, L. J., \& Wilson, M. R. (2013a). Quiet eye and choking: Online control breaks down at the point of performance failure. Medicine \& Science in Sports \& Exercise. doi:10.1249/MSS.0b013e31829406c7

Vine, S. J., Moore, L. J., \& Wilson, M. R. (2013b). Quiet eye training: The acquisition, refinement and resilient performance of targeting skills. European Journal of Sport Science. doi:10.1080/ 17461391.2012 .683815

Wilson, M. R., \& Pearcey, R. (2009). On the right line: The visuomotor control of straight and breaking golf putts. Perceptual and Motor Skills, 109, 555-562. doi:10.2466/ pms.109.2.555-562

Witt, J. K., Linkenauger, S. A., \& Proffitt, D. R. (2012). Get me out of this slump! visual illusions improve sports performance. Psychological Science, 23, 397-399. doi:10.1177/0956797611428810 